\title{
The roughness calculation of the basal boundary for the ice-sounding data collected at Princess Elizabeth Land (PEL)
}

\author{
Shinan Lang ${ }^{1 *}$, Xiangbin $\mathrm{Cui}^{2}$, and Ben $\mathrm{Xu}^{1,2}$ \\ ${ }^{1}$ Beijing University of Technology, Beijing 100124, China \\ ${ }^{2}$ Polar Research Institute of China, Shanghai 200136, China
}

\begin{abstract}
In this paper, we calculated the roughness of the basal boundary collected at Princess Elizabeth Land (PEL) to evaluate the topographic structure via the ice-sounding data collected during $32^{\text {nd }}$ and $33^{\text {rd }}$ Chinese Antarctic Research Expeditions (CHINARE 32 and 33). The calculation is achieved by a two-parameter roughness index method, which could differentiate different classes of subglacial landscape, in particular between erosional and depositional settings. Finally, the calculation results of partial regions of PEL are illustrated to describe the roughness of the detected regions.
\end{abstract}

\section{Introduction}

China has conducted Antarctic research expeditions for over thirty years, primarily using ground-based methods as the main source of scientific observations [1-5]. This has led to limited spatial data coverage and data volume. The first fixed-wing airborne platform of the Chinese Antarctic expeditions, named Snow Eagle 601, was instrumented with ice sounding radar (ISR), gravimeter, magnetometer, laser altimeter and optical camera. The aircraft was modified in 2015 and applied to geophysical investigation of Princess Elizabeth Land (PEL) during the $32^{\text {nd }}$ and $33^{\text {rd }}$ Chinese National Antarctic Research Expeditions (CHINARE 32 and 33). The capabilities of the aircraft and instrument suite were fully demonstrated with $\sim 30,000 \mathrm{~km}$ of data collected over high interest targets. The survey covered an area of PEL in East Antarctica, as shown in Fig. 1, where a large geophysical data gap exists. The fan-shaped and radial pattern flight lines extending from the airfield near the coastal Zhongshan Station to the interior of the East Antarctic Ice Sheet and was designed to maximize aero-geophysical coverage as well as to intersect with historical ICECAP and AGAP surveys wherever possible. The investigation focused on mapping subglacial topography in this unknown region, interpreting ice sheet evolution over PEL, surveying a proposed subglacial lake (potentially the second longest in Antarctica), identifying subglacial channels and canyons that possibly connect to the coast, as well as searching for the oldest ice. Overall, 18 flights were completed in addition to test flights, with a total length of the lines surveyed of $\sim 32,000 \mathrm{~km} \mathrm{[6].}$

\footnotetext{
*Corresponding author: langshinan@,bjut.edu.cn
} 


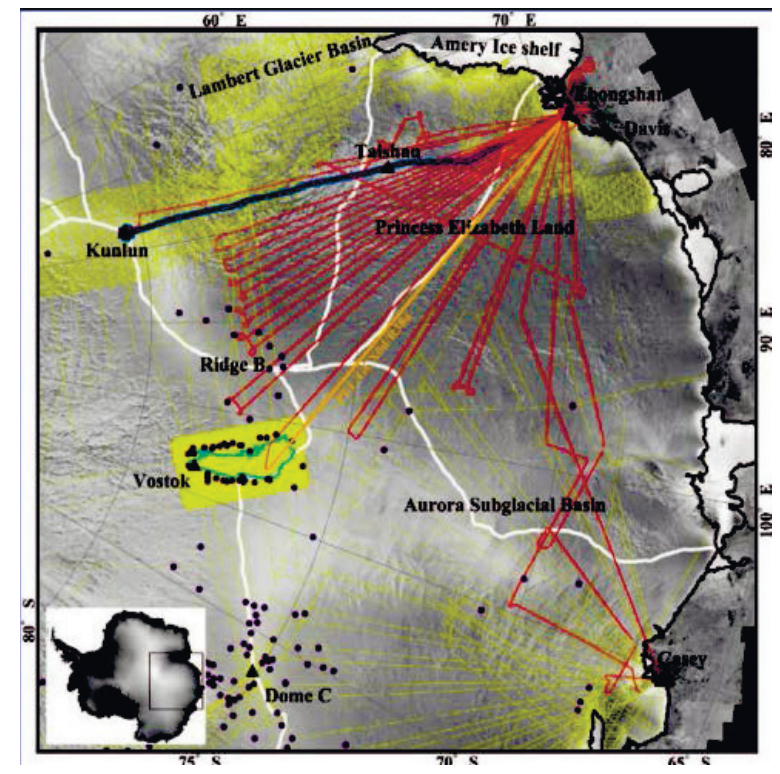

Fig. 1. The survey covered areas of PEL in East Antarctica.

The roughness calculation of the basal boundary is the first step we set to analysis the topographic structure. The method we utilized is called a two-parameter roughness index method [7]. This method derived a two parameter Fourier transformation (FT) roughness index $\{\xi, \eta\}$, where $\xi$ is based on the FT of elevation, and $\eta$ is based on both the FT of elevation and bed-slope profile. Hence, the index $\{\xi, \eta\}$ effectively characterizes the vertical and horizontal roughness. Firstly, we obtain the echograms of the ice-sounding radar data via two-dimensional focused synthetic aperture radar (SAR) imaging algorithm. Afterwards, the air-ice interface and the basal interface are picked by manual operation. Finally, the roughness calculation is realized by the above-mentioned index to evaluate the topographic structure. The calculated results are demonstrated in the Antarctic map to indicate the corresponding topographic relief.

\section{Calculating procedures}

The whole calculating procedures mainly include three parts: ice-sounding radar data imaging, air-ice interface and the basal interface pick, and roughness calculation. The details of each part will be introduced in the following sections.

\subsection{Ice-sounding radar data imaging}

The two-dimensional focused SAR algorithm is achieved by correlating the signal with the reference function, which is given by

$$
\mathfrak{R}_{v m}(x, \hat{t})=\int_{T} \int_{L} v\left(x+x^{\prime}, \hat{t}+\tau\right) m^{*}\left(x^{\prime}, \tau\right) d x^{\prime} d \tau
$$

where $L$ is the synthetic aperture length and the period $T$ is the measure of arrival times for a point target for the given synthetic aperture length, as shown in Fig. 2. The simplified flowchart of this algorithm is illustrated in Fig. 3. 


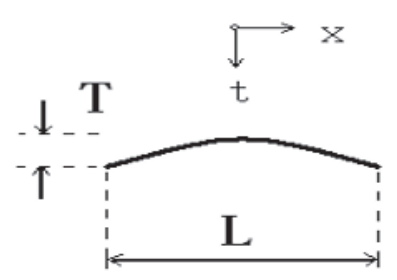

Fig. 2. The sketch map of synthetic aperture length and the period.

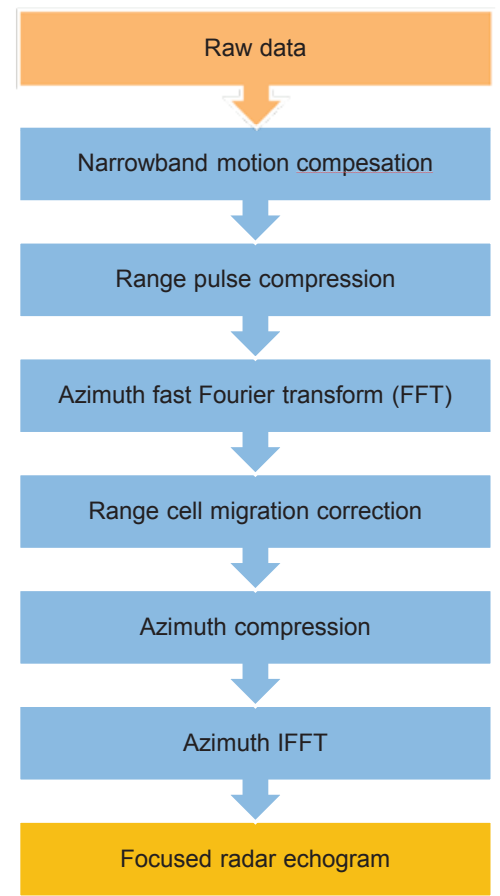

Fig. 3. The flowchart of two-dimensional focused SAR algorithm.

\subsection{Interface pick}

After the echogram of ice-sounding radar has been achieved, the air-ice interface and the basal interface are picked by manual operation. We designed an strategy based on cubic spline interpolation to promote the precision of the picked result. The strategy is implemented as follows: 1) the upper surface of the air-ice/basal interface is picked by manual operation; 2) the lower surface of the air-ice/basal interface is also picked by manual operation; 3) the cubic spline interpolation is realized between the above-picked upper and lower surfaces. An sample of the picked result is given in Fig. 4. 


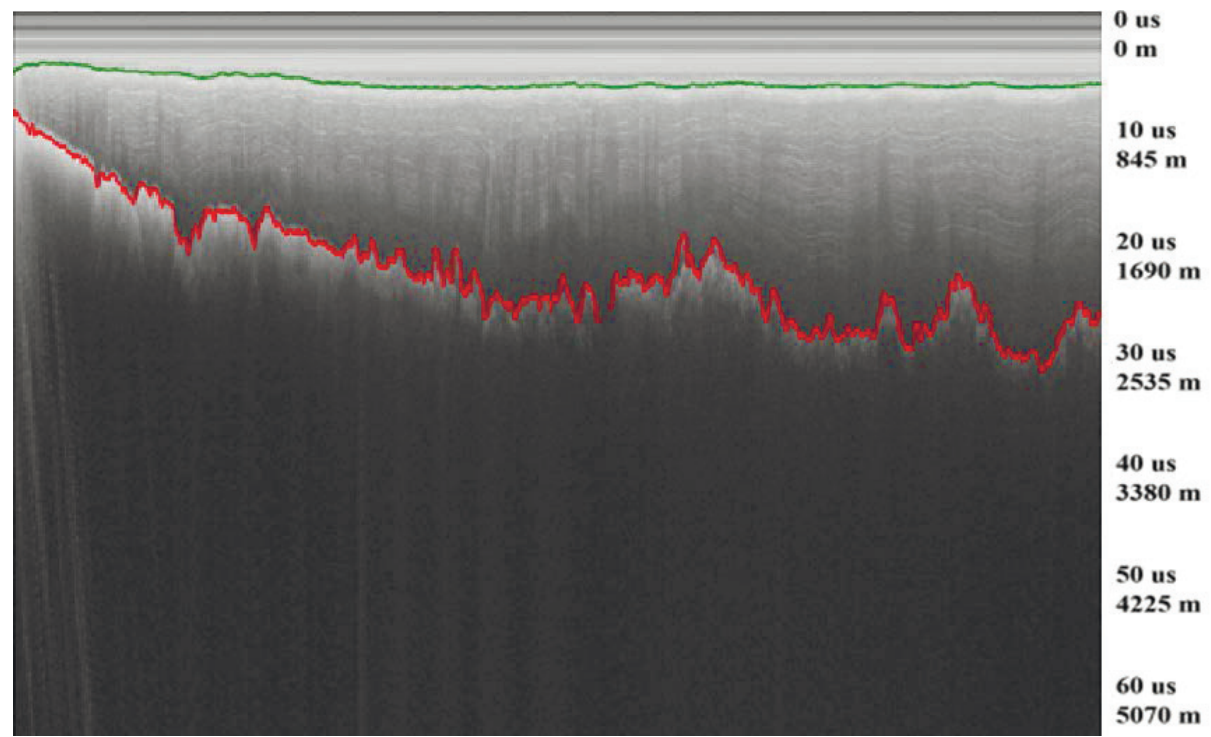

Fig. 4. An sample of the picked result.

\subsection{Roughness calculation}

The method we utilized to calculate roughness called a two-parameter roughness index method [7]. This method derived a two parameter Fourier transformation (FT) roughness index $\{\xi, \eta\}$, where $\xi$ is based on the FT of elevation, and $\eta$ is based on both the FT of elevation and bed-slope profile. The parameter $\xi$ is obtained via calculating the intergration of the spectral power density of elevation computed by above-picked interfaces. The parameter $\eta$ is achieved by calculating the bed slope under certain elevation variation in along-track direction. The equations for $\{\xi, \eta\}$ computing is given by

$$
\xi=\int_{k_{1}}^{k_{2}} S(k) d k
$$

where $k_{1}$ is zero, $k_{2}$ is infinity, and $S(k)$ is the spectral power density of elevation.

$$
\eta=\frac{\xi}{\xi_{s l}}=\frac{\int_{k_{1}}^{k_{2}} S(k) d k}{\int_{k_{1}}^{k_{2}} S_{s l}(k) d k}
$$

where $S(k)$ and $S_{s l}(k)$ both indicate the elevation variation under a fixed along-track distance.

The meanings of $\{\xi, \eta\}$ could be explained as follows:

$>$ Low $\xi$, high $\eta$ : dominated by deposition reflecting marine landscapes.

$>$ High $\xi$, low $\eta$ : characteristic of beds which are alpine in nature yet cold-based so that no subglacial sliding occurs.

$>$ High $\xi$, high $\eta$ : a continental settings which have undergone deposition over a short period and have subsequently become protected beneath a cold and slowflowing basal thermal regime.

$>$ Low $\xi$, low $\eta$ : a continental setting influenced by intensive erosion. 


\section{Field deployment results}

We calculate the roughness of the basal boundary collected at PEL o evaluate the topographic structure via the ice-sounding data collected during CHINARE 32 and 33. The calculation is achieved via the roughness index method. The calculation results of partial regions of PEL are illustrated in Fig. 5.

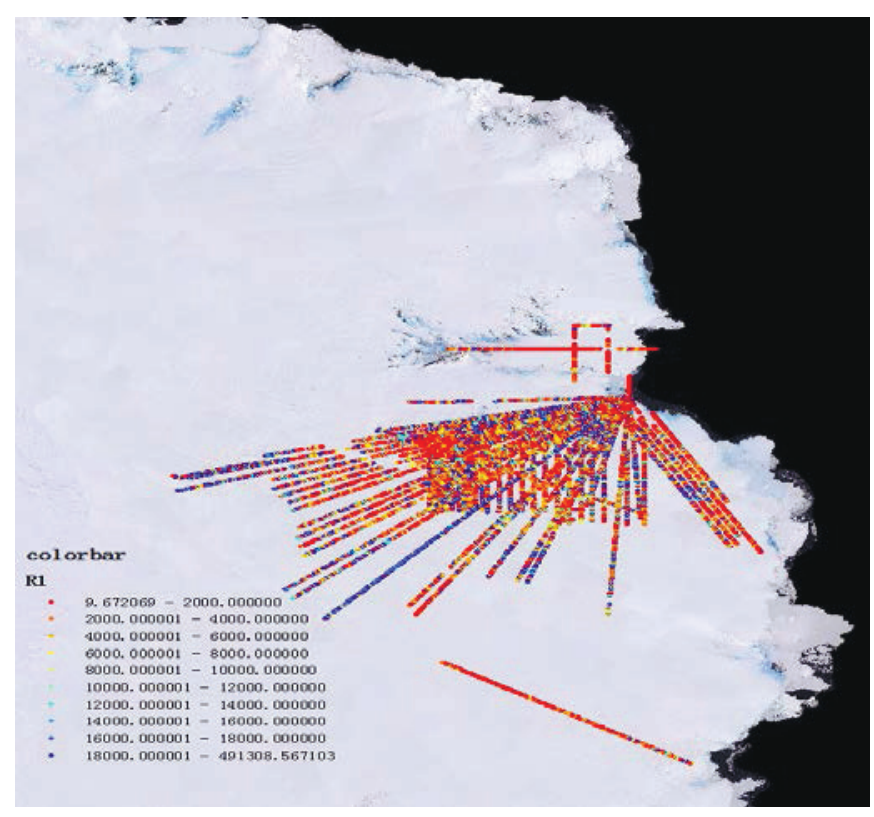

(a) Result of parameter $\xi$

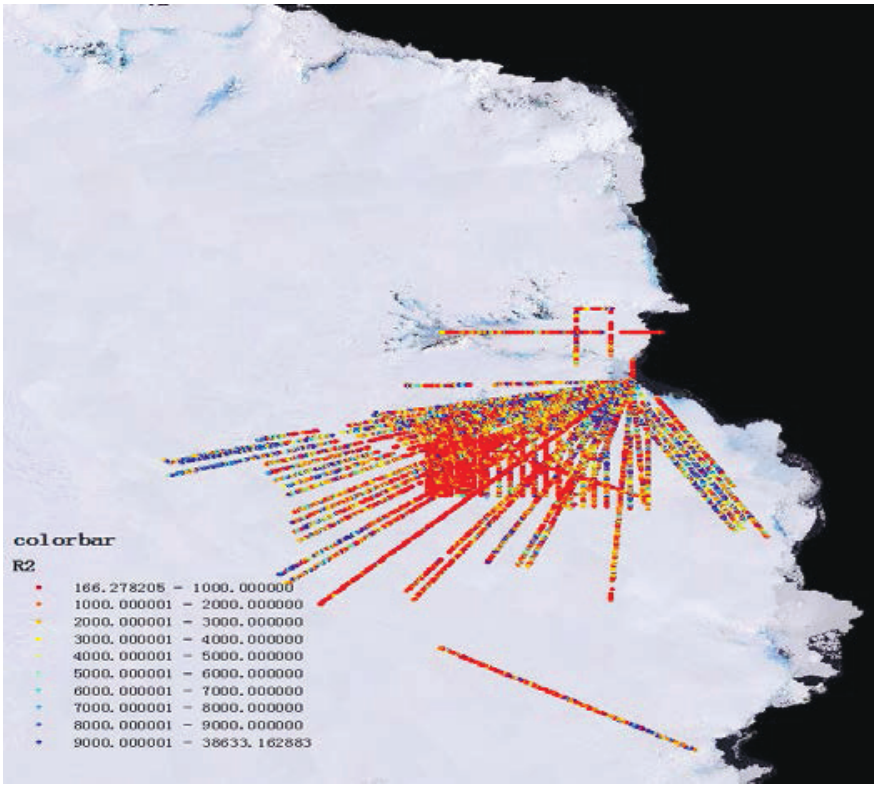

(b) Result of parameter $\eta$

Fig. 5. Roughness calculation result. 


\section{References}

1. Ren. J., D. Qin, and C. Xiao, J. Glaciol. Geocryol 23, 1 (2001)

2. Shengkai, Z., Dongchen, E., Zemin, W., Yuansheng, L., Bo, J., Chunxia, Z., Ann. Glaciol. 48 (2008)

3. Cui, X., Sun, B., Tian, G., Tang, X., Zhang, X., Jiang, Y., Li, X, Chi. Sci. Bul. 55, 4-5 (2010)

4. Minghu, D., Cunde, X., Yuansheng, L., Jiawen, R., Shugui, H., Bo, J., Bo, S., J. Glaciol. 57, 204 (2011)

5. Yang, Y., Sun, B., Wang, Z., Ding, M., Hwang, C., Ai, S., Dongchen, E., J. Glaciol. 60, $222(2014)$

6. Cui, X., Greenbaum, J. S., Beem, L. H., Guo, J., Ng, G., Li, L., Sun, B., J. Env. Eng. Geophys. 23, 1 (2018)

7. Li, X., Sun, B., Siegert, M. J., Bingham, R. G., Tang, X., Zhang, D., ... \& Zhang, X., J. Glaciol. 56, 199 (2010) 